\title{
Perioperative Risk Factors Predisposing to Atrial Fibrillation After CABG Surgery
}

\author{
Alaa Omar, MD, Ehab El-Shihy, MD, Mahmoud Singer, MD, David Zarif, MSSC, Omar Dawoud, MD \\ Department of Cardiothoracic Surgery, Faculty of Medicine, Cairo University, Giza, Egypt
}

\section{ABSTRACT}

Objectives: To detect perioperative risk factors for atrial fibrillation $(\mathrm{AF})$ after coronary artery bypass graft (CABG) and to assess the impact of $\mathrm{AF}$ on outcome and postoperative complications.

Methods: We undertook a prospective observational study of 1000 consecutive patients who underwent isolated CABG in Cairo University hospitals and other centers from March 2019 to November 2020. Patients were subsequently divided into 2 groups depending on the occurrence of postoperative AF. Preoperative, intraoperative, and postoperative risk factors were recorded for all patients, as well as postoperative mortality, complications, and hospital and intensive care unit (ICU) lengths of stay.

Results: Postoperative atrial fibrillation $(\mathrm{POAF})$ occurred in 78 patients $(7.8 \%)$, with significant risk factors of age $(P=.001)$, low ejection fraction $(P=.001)$, absence of preoperative betablocker use $(P=.001)$, and presence of right coronary artery lesion $(P=.003)$. The intraoperative significant risk factor was the absence of total coronary revascularization $(P=.001)$. Postoperative significant risk factors were electrolyte imbalance $(P=.001)$ and postoperative inotropes $(P=.02)$. Patients with postoperative AF had increased risk of mortality $(P=.001)$ and longer ICU $(P=.001)$ and hospital $(P=.001)$ stays.

Conclusion: The risk of POAF can be decreased by modifying perioperative adjustable risk factors, namely routinely using preoperative beta-blockers (unless contraindicated), achieving total coronary revascularization, avoiding postoperative electrolyte imbalance, and avoiding unnecessary use of inotropic support.

\section{INTRODUCTION}

Atrial fibrillation (AF) is a supraventricular tachyarrhythmia with uncoordinated atrial activity predisposing to deterioration in cardiac mechanical activity [Maisel 2001]. Postoperative atrial fibrillation (POAF) may be a temporary, self-limiting phenomenon, but it can lead to poor

Received March 3, 2021; accepted March 11, 2021.

Correspondence: Alaa Omar, MD, Cairo University, 14 Mossadak st. al-dokki, 6th Floor, Cairo, Egypt; (e-mail: alaaomarcts1@yahoo.com). hemodynamics with jeopardized myocardial function and prolonged intensive care unit (ICU) and hospital stays. POAF has a multifactorial pathophysiology that has not been fully explained; multiple perioperative factors may show a proarrhythmogenic effect, increasing the incidence of POAF [Echahidi 2008]. Incidence of AF after coronary artery bypass graft (CABG) ranges from $20 \%$ to $40 \%$, with the highest recorded incidence on postoperative day 2 [Aranki 1996]. Post-CABG AF is linked to progressive deleterious effects such as congestive heart failure, prolonged ICU and hospital stays, increased health system costs, increased hospital mortality, and poor survival [Filardo 2009].

\section{METHODS}

This research was performed after informed consent was obtained from each patient. We undertook a prospective observational study on a group of 1000 consecutive patients who underwent isolated CABG in Cairo University hospitals and other centers from March 2019 to November 2020. Inclusion criteria included any patient undergoing isolated CABG with preoperative sinus rhythm. We excluded patients requiring any associated valvular intervention, those with preoperative $\mathrm{AF}$, and patients on antiarrhythmic drugs other than beta-blockers. There were some significant differences in demographic data and baseline clinical characteristics between the groups (Tables 1 and 2).

In all cases, medical history, physical examination, complete laboratory investigation, chest $\mathrm{x}$-rays, $2 \mathrm{D}$ and $\mathrm{M}$ mode echocardiography (ECG), and coronary angiography were evaluated. Recorded preoperative characteristics included

Table 1. Demographic Data of the 2 Groups*

\begin{tabular}{|c|c|c|c|}
\hline Characteristic & Non-AF $(n=922)$ & $\mathrm{AF}(\mathrm{n}=78)$ & $P$ Value \\
\hline Age $(y)$ & $57.07 \pm 9.16$ & $67.51 \pm 6.80$ & $.001 \dagger$ \\
\hline \multicolumn{4}{|l|}{ Sex } \\
\hline Female & $150(16.3)$ & $17(21.8)$ & .209 \\
\hline Male & 772 (83.7) & $61(78.2)$ & \\
\hline
\end{tabular}

*Data are mean \pm SD or $n(\%)$.

$\dagger$ Significant at $P<.05$. 
age, sex, hypertension, diabetes, preoperative use of betablockers, smoking, preoperative ejection fraction (EF), left atrium size, and presence of a significant right coronary artery lesion. Intraoperative parameters included total revascularization, cross-clamp time, total cardiopulmonary bypass (CPB) time, and use of intra-aortic balloon pump. All patients were assessed postoperatively to determine the use of inotropes and the occurrence of electrolyte imbalance. Postoperative ICU and hospital lengths of stay, mortality, reoperation for bleeding, and wound infection were recorded.

Routine median sternotomy incision was performed in all cases. Standard CPB was initiated through aorto-atrial cannulation in all patients after ensuring that the activated clotting time was $>480$ seconds. The left internal mammary artery was almost always grafted to the left anterior descending artery, and the great saphenous vein was used to graft other targets. Warm intermittent antegrade blood/potassium cardioplegia was used for myocardial protection. At the end of $\mathrm{CPB}$, heparin was neutralized with protamine sulfate. In off-pump CABG, half the dose of heparin was used. First performed was the anastomosis of left internal mammary artery to left anterior descending artery, followed by other grafts, and then proximal vessels were connected to the aorta. Surgery was followed in both techniques by hemostasis and routine closure.

Table 2. Preoperative Parameters in the 2 Groups*

\begin{tabular}{lccc}
\hline Factor & Non-AF $(\mathrm{n}=922)$ & AF $(\mathrm{n}=78)$ & $P$ Value \\
\hline Smoking & $470(51.0)$ & $45(57.7)$ & .254 \\
Diabetes & $475(51.5)$ & $48(61.5)$ & .089 \\
Hypertension & $598(64.9)$ & $43(55.1)$ & .085 \\
Preoperative beta-blocker use & $831(90.1)$ & $55(70.5)$ & $.001 \dagger$ \\
Right coronary lesion & $688(74.6)$ & $70(89.7)$ & $.003 \dagger$ \\
EF & & $25.0,69.0$ & $.001 \dagger$ \\
$\quad$ Minimum, maximum & $25.0,75.0$ & $50.33 \pm 11.32$ & .222 \\
$\quad$ Mean \pm SD & $56.21 \pm 11.01$ & $3.5,4.5$ \\
Left atrium size (cm) & & $4.00 \pm 0.25$ \\
$\quad$ Minimum, maximum & $2.6,5$ & \\
$\quad$ Mean \pm SD & $3.94 \pm 0.43$ & \\
\hline
\end{tabular}

*Data are mean \pm SD or $\mathrm{n}(\%)$ unless noted otherwise.

$\dagger$ Significant at $P<.05$.

Table 3. Intraoperative Parameters in the 2 Groups*

\begin{tabular}{llll}
\hline Parameter & Non-AF $(\mathrm{n}=922)$ & $\mathrm{AF}(\mathrm{n}=78)$ & $P$ Value \\
\hline Total revascularization & $865(93.8)$ & $65(83.3)$ & $.001 \dagger$ \\
On pump & $813(88.2)$ & $64(82.1)$ & .114 \\
Off pump & $109(11.8)$ & $14(17.9)$ & .114 \\
Total CPB (min) & & & .180 \\
$\quad$ Minimum, maximum & $30.0,180.0$ & $65.0,135.0$ & .134 \\
$\quad$ Mean \pm SD & $101.94 \pm 27.56$ & $106.64 \pm 18.32$ & .710 \\
Cross-clamp time (min) & & & $35.0,120.0$ \\
$\quad$ Minimum, maximum & $20.0,120.0$ & $70.16 \pm 15.01$ & $1(1.3)$ \\
$\quad$ Mean \pm SD & $66.52 \pm 18.95$ & & \\
Intra-aortic balloon & $8(0.9)$ & & \\
\hline
\end{tabular}

*Data are mean \pm SD or $\mathrm{n}(\%)$ unless noted otherwise.

$\dagger$ Significant at $P<.05$. 
AF was diagnosed on the basis of abnormalities on ECG lasting for $\geq 30$ seconds and characterized by irregular RR intervals, absent $\mathrm{P}$ waves, and different intervals between atrial contractions (cycle $<200 \mathrm{~ms}$ ).

Results were expressed as mean \pm standard deviation (SD) or $\mathrm{n}(\%)$. Comparisons between categorical data were performed using $\chi^{2}$ test or Fisher's exact test if cell count was $<5$. Tests of normality (Kolmogorov-Smirnov) were used to measure the distribution of data. Multivariate analysis was used to study the predictive effect of different variables for developing AF. Statistical Package for Social Sciences software (version 20 for Windows) was used for data analysis. $P$ values $\leq .05$ were considered significant.

\section{RESULTS}

Results are presented in 2 groups based on the occurrence of POAF: the non-AF group included 922 patients, and the AF group, 78 patients. Demographic data are shown in Table 1. The mean age was significantly higher in the AF group (67.5 versus 57.07 years; $P<.001)$.

Preoperative parameters were analyzed for both groups. In the non-AF group, 831 patients $(90.1 \%)$ received preoperative beta-blockers, compared with 55 patients $(70.5 \%)$ in the $\mathrm{AF}$ group $(P=.001)$. The presence of a significant right coronary artery lesion was also more common in the
AF group, and was found in 70 patients (89.7\%) versus 688 $(74.6 \%)$ in the non-AF group. Another statistically significant factor was preoperative EF, which was higher in the non-AF group (mean 56.2\%) than the AF group (50.3\%). Preoperative parameters for both groups are summarized in Table 2.

Intraoperative parameters, including choice of surgical technique (on or off pump), bypass time, cross-clamp time (for on-pump cases), and the application of intra-aortic balloon pump, showed no statistically significant effect on the occurrence of POAF. The only intraoperative variable showing an effect was total coronary revascularization, which was done in $93.8 \%$ of cases in the non-AF group compared with $83.3 \%$ in the AF group. Preoperative parameters for both groups are summarized in Table 3 .

Postoperatively, a higher percentage of patients in the AF group were on inotropes $(56.4 \%$ versus $42.6 \%$ in the non-AF group), with a higher mean dose of adrenaline. Also, the presence of electrolyte disturbance was higher in the AF group (34.6\% versus $0.7 \%$ in the non-AF group). Mortality was higher in the AF group, as were the mean ICU and hospital lengths of stay. No statistically significant difference was observed regarding postoperative bleeding or wound infection between the 2 groups. Postoperative parameters are summarized in Table 4.

In the AF group, 47 patients $(60.25 \%)$ had 1 episode of $\mathrm{AF}$, and 31 (39.7\%) had 2 episodes. In the 78 patients who developed AF, only 1 (1.28\%) needed direct-current shock to

Table 4. Postoperative Risk Factors and Outcomes in the 2 Groups*

\begin{tabular}{|c|c|c|c|}
\hline Inotropes & $395(42.8)$ & $44(56.4)$ & $.02 \dagger$ \\
\hline Adrenaline dose $(\mathrm{ng} / \mathrm{kg} / \mathrm{min})$ & & & $.001 \dagger$ \\
\hline Range & 20.0 to 200.0 & 50.0 to 150.0 & \\
\hline Dobutamine & $2(0.2)$ & $1(1.3)$ & .099 \\
\hline Dopamine & $5(0.5)$ & $0(0.0)$ & .514 \\
\hline Electrolyte imbalance & $6(0.7)$ & $27(34.6)$ & $.001 \dagger$ \\
\hline Bleeding & $12(1.3)$ & $1(1.3)$ & 1.000 \\
\hline Wound infection & $26(2.82)$ & $4(5.13)$ & .251 \\
\hline Hospital stay $(d)$ & & & $.001 \dagger$ \\
\hline Minimum, maximum & $4.0,13.0$ & $5.0,14.0$ & \\
\hline Mean \pm SD & $5.31 \pm 0.95$ & $7.33 \pm 1.51$ & \\
\hline Mortality & $1(0.1)$ & $2(2.6)$ & $.001 \dagger$ \\
\hline
\end{tabular}

*Data are mean \pm SD or $\mathrm{n}(\%)$ unless noted otherwise.

†Significant at $P<.05$. 
return to sinus rhythm; 6 patients $(7.69 \%)$ returned to sinus rhythm after correction of electrolyte imbalance and other metabolic factors; and 71 patients (91\%) needed medical cardioversion using amiodarone (2.5 to $5 \mathrm{mg} / \mathrm{kg}$ intravenously over 20 minutes; then $15 \mathrm{mg} / \mathrm{kg}$ or $1.2 \mathrm{~g}$ intravenously over 24 hours; then continuation orally at $200 \mathrm{mg} /$ day). The highest incidence of POAF was on postoperative day 2 . The timing of POAF is summarized in Figure 1.

\section{DISCUSSION}

Atrial fibrillation is the most common arrhythmia that occurs after cardiac surgical procedures. It may have clinical consequences for postoperative patients, and it may affect hospital stays and costs [Aranki 1996]. Advances in operative technique and postoperative care over the years have not decreased the occurrence of this significant complication [Gorczyca 2018]. Our study conducted on 1000 patients shows that the incidence of $\mathrm{AF}$ after isolated $\mathrm{CABG}$ was only $7.8 \%$, in contrast with other studies that showed a higher incidence $(16.9 \%$ in the retrospective study by LaPar [2014] including 40,585 patients; $23.2 \%$ in a study conducted by Banach [2006] on 1200 patients, including some with a history of supraventricular arrhythmia, who were excluded in our study). This difference can also be explained by the younger mean age in our study, the frequent use of preoperative beta-blockers, the high incidence of total revascularization, the low incidence of the use of postoperative inotropes, and the low incidence of postoperative electrolyte imbalance.

Regarding demographics, age was a statistically significant factor increasing the incidence of POAF (non-AF age $57.07 \pm$ 9.16 years compared with $67.51 \pm 6.80$ years in the AF group; $\mathrm{P}<.001)$. These results are similar to those of Ismail [2017], Lee [2018], and Filardo [2009], all of whom showed increased incidence of POAF in higher age groups. The higher overall mean age in those studies can explain the difference in the overall incidence of AF between our study and theirs. The higher incidence of $\mathrm{AF}$ with age may be related to associated comorbidities as well as degenerative changes in the atrial wall [Aranki 1996].

Patients receiving preoperative beta-blockers were found to have a lower incidence of POAF in our study. The study by Lee and Jang [2018] showed no significant difference, but with a low overall percentage of patients receiving preoperative beta-blockers, which may explain the difference in the incidence of AF between the 2 studies: in their work, only $56.82 \%$ received beta-blockers in the non-AF group and $53.27 \%$ in the AF group. Our study showed statistical significance regarding preoperative EF $(P=.001)$, with lower EF a predisposing factor for $\mathrm{AF}$. In the non-AF group, the mean EF was $(56.21 \% \pm 11.01 \%)$, and it was $(50.33 \% \pm 11.32 \%)$ in the AF group. Kannell [1982] reported increased incidence of $\mathrm{AF}$ with decreased left ventricular contractility in the general population.

Choice of surgical technique (on pump versus off pump) and total bypass and cross-clamp times did not affect the incidence of POAF. Our study showed statistical significance

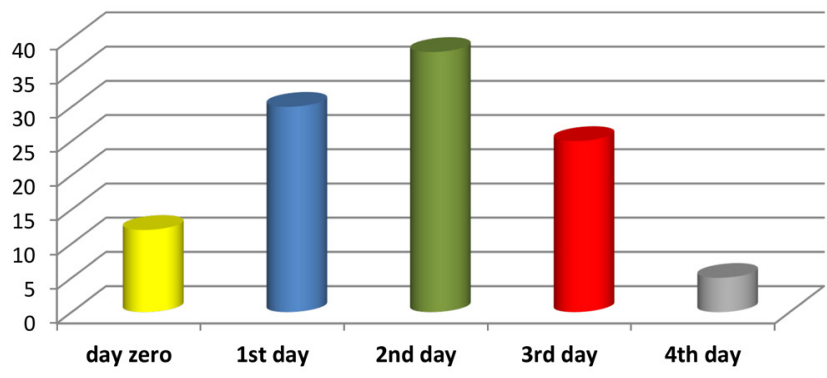

Figure 1. Timing of AF.

regarding total revascularization as a preventive factor for POAF $(P=.001)$ : in the non-AF group, $93.8 \%$ were totally revascularized in comparison with $83.3 \%$ in the $\mathrm{AF}$ group.

Postoperatively, electrolyte disturbance and inotropic support, namely adrenaline, were shown to be significant risk factors in the development of POAF. Potassium plays a central role in cardiac electrophysiology. Serum K+ concentrations are commonly low after cardiac surgery [Polderman 2004]. Hypokalemia (serum potassium $<3.5 \mathrm{mmol} / \mathrm{l}$ ) leads to cellular hyperpolarity, higher resting potential, increased automaticity and excitability, and ventricular arrhythmias [January 2014]. The administration of adrenergic drugs is an independent risk factor for AF after CABG. Studies have suggested that the increased sympathetic response enhances the development of POAF [Salaria 2005].

The occurrence of POAF was associated with higher early mortality in our study $(0.1 \%$ in the non-AF group and $2.6 \%$ in the AF group). Hospital and ICU stay were also prolonged in the AF group, a finding shared by Banach [2006].

\section{CONCLUSION}

Postoperative $\mathrm{AF}$ is a common complication after $\mathrm{CABG}$ surgery, increasing mortality, as well as ICU and hospital lengths of stay. The risk of postoperative $\mathrm{AF}$ can be decreased by modifying perioperative adjustable risk factors, including routinely using preoperative beta-blockers (unless contraindicated), achieving total coronary revascularization, avoiding postoperative electrolyte imbalance, and avoiding unnecessary use of inotropic support.

\section{REFERENCES}

Aranki SF, Shaw DP, Adams DH, Rizzo RJ, Couper GS, VanderVliet M, Collins JJ, Cohn LH, Burstin HR. Predictors of atrial fibrillation after coronary artery surgery. Circulation 1996;94:390-397.

Banach M, Rysz J, Drozdz J, Okonski P, Misztal M, Barylski M, et al. Risk factors of atrial fibrillation following coronary artery bypass grafting: A preliminary report. Circ J 2006;70:438-441.

Echahidi N, Pibarot P, O'Hara G, et al. Mechanisms, prevention, and treatment of atrial fibrillation after cardiac surgery. J Am Coll Cardiol 2008;51:793-801. 
Filardo G, Hamilton C, Hebeler RF, Hamman B, Grayburn P. Newonset postoperative atrial fibrillation after isolated coronary artery bypass graft surgery and long-term survival. Circ Cardiovasc Qual Outcomes 2009;2:164-169.

Gorczyca I, Michta K, Pietrzyk E, Wożakowska-Kapłon B. Predictors of post-operative atrial fibrillation in patients undergoing isolated coronary artery bypass grafting. Kardiol Pol 2018;76:195-201.

Ismail MF, El-mahrouk AF, Hamouda TH, Radwan H, Haneef A, Jamjoom AA. Factors influencing postoperative atrial fibrillation in patients undergoing on-pump coronary artery bypass grafting, single center experience. J Cardiothorac Surg 2017;12:1-7.

January CT, Wann LS, Alpert JS, Calkins H, Cigarroa JE, Cleveland JC, et al. AHA/ACC/HRS guideline for the management of patients with atrial fibrillation: A report of the American College of Cardiology/American Heart Association Task Force on Practice Guidelines and the Heart Rhythm Society. J Am Coll Cardiol 2014;64:e1-e76.
Kannell WB, Abbott RD, Savage DD, McNamara PM. Epidemiologic features of chronic atrial fibrillation: The Framingham Study. N Engl J Med 1982;306:1018-1022.

Lapar DJ, Speir AM, Crosby IK, Fonner E, Brown M, Rich JB, et al. Postoperative atrial fibrillation significantly increases mortality, hospital readmission, and hospital costs. Ann Thorac Surg 2014;98:527-533.

Lee J, Jang I. Predictors affecting postoperative atrial fibrillation in patients after coronary artery bypass graft. Clin Nurs Res 2020;29:543-550.

Maisel WH, Rawn JD, Stevenson WG. Atrial fibrillation after cardiac surgery. Ann Intern Med 2001;135:1061-1073.

Polderman KH, Girbes ARJ. Severe electrolyte disorders following cardiac surgery: A prospective controlled observational study. Crit Care 2004;8:R459-R466.

Salaria V, Mehta NJ, Abdul-Aziz S, Mohiuddin SM, Khan IA. Role of postoperative use of adrenergic drugs in occurrence of atrial fibrillation after cardiac surgery. Clin Cardiol 2005;28:131-135. 